\title{
The consequences of parity symmetry for higher-order statistics of cosmic shear and other polar fields
}

\author{
P. Schneider ${ }^{\star}$ \\ Institut für Astrophysik und Extraterrestrische Forschung, Universität Bonn, Auf dem Hügel 71, 53121 Bonn, Germany
}

Received 7 May 2003 / Accepted 30 June 2003

\begin{abstract}
We consider the parity transformation and the consequences of parity invariance on the $n$-point correlation function of the cosmic shear caused by gravitational lensing by the large-scale structure, or any other polar (or "spin-2") field. The decomposition of the shear field into $E$ - and $B$-modes then yields the result that any correlation function which contains an odd number of $B$-mode shear components vanishes for parity-invariant random shear fields. In particular, this result implies that the expectation value of the third-order cross aperture statistics, $\left\langle M_{\times}^{3}\right\rangle$, vanishes for parity-invariant shear fields. Therefore, a significant detection of a non-zero value of $\left\langle M_{\times}^{3}\right\rangle$ in a cosmic shear survey does not indicate the presence of $B$-modes, but of an underestimate of the statistical uncertainty or cosmic variance, or a remaining systematic effect in the shear measurement. We argue that the parity invariance provides a very specific diagnostic of systematic effects in shear data. Our results apply as well to the linear polarization of the cosmic microwave background.
\end{abstract}

Key words. dark matter - methods: statistical - cosmology: miscellaneous - cosmic microwave background

\section{Introduction}

Cosmic shear, resulting from the gravitational deflection of light bundles from distant sources by the tidal gravitational field of the large-scale structure, has been recognized as an important tool for observational cosmology (e.g., Blandford et al. 1991; Miralda-Escudé 1991; Kaiser 1992; see Mellier 1999 and Bartelmann \& Schneider 2001 for recent reviews). It allows the direct investigation of the (dark) matter distribution up to redshifts $\sim 1$, without making assumptions about the relation between dark matter and luminous tracers, like galaxies. Therefore, since the first detections of cosmic shear (Bacon et al. 2000; Kaiser et al. 2000; van Waerbeke et al. 2000; Wittman et al. 2000) intensive observational and theoretical research has been performed.

Most of the work up to now has concentrated on secondorder statistics of the shear field. However, Bernardeau et al. (2002) have detected a significant third-order shear signal in the Virmos-Descart cosmic shear survey. It was realized before that the third-order cosmic shear statistics would provide an invaluable tool for cosmological parameter estimates; in particular, a third-order shear measurement breaks the (near) degeneracy between the density parameter $\Omega_{m}$ and the power spectrum normalization $\sigma_{8}$ (Bernardeau et al. 1997; Jain \& Seljak 1997; van Waerbeke et al. 1999). These theoretical studies concentrated on the third-order statistics of the convergence field, from which the shear field is derived. However, as the shear is

\footnotetext{
* e-mail: peter@astro.uni-bonn.de
}

the observable (or more precisely, the observable galaxy ellipticities provide an unbiased estimate of the shear), not the convergence, recent interest has focused on determining the thirdorder statistical properties of the shear itself.

Since the shear is a two-component quantity, the three-point correlation function (3PCF) has eight independent components and depends on three arguments. Bernardeau et al. (2003) considered a specific combination of components of the 3PCF, calculated its properties, both analytically and numerically, and applied this to measure the 3PCF on the Virmos-Descart data (Bernardeau et al. 2002). With the large number of components of the 3PCF, it is not clear a priori which of them carry most of the information about the underlying matter field. In contrast to the two-point correlation function (2PCF), which has only two non-vanishing components (the other two vanish by parity invariance), none of the eight components of the 3PCF vanishes for a general set of three points. Only for specific triangles (where two or three sides are equal) does parity imply that some components vanish (Schneider \& Lombardi 2003; hereafter SL03). This has later been verified using ray-tracing simulations through a cosmological matter distribution, as well as with analytic estimates of the 3PCF from the halo model (Takada \& Jain 2003a; Zaldarriaga \& Scoccimarro 2003). As it turned out, investigating the statistical properties of the $3 \mathrm{PCF}$ of a polar field is far from trivial, and some confusion about symmetry properties has arisen.

The purpose of this paper is to clarify some of the issues related to parity transformations of the shear field. In Sect. 2 
we recall the definition of the shear 3PCF and its behavior under parity transformations. In Sect. 3 the decomposition of a general shear field into $E$ - and $B$-modes (Crittenden et al. 2002; Schneider et al. 2002) is introduced. The central result of this paper, derived in Sect. 4 , is as follows: for a pure $B$-mode shear field whose statistical properties are invariant under parity transformation (hereafter: parity symmetric), the 3PCF vanishes identically. More generally, the $n$-point shear correlation function containing $m B$-mode shear components and $(n-m)$ $E$-mode shear components vanishes identically for $m$ odd. This result will be shown both by an explicit calculation, as well as by a simple heuristic, though accurate, consideration (at the beginning of Sect. 4).

\section{Parity transformation of the 3PCF}

\subsection{Definition of the 3PCF}

We consider a shear field (or more generally, any polar field) that is statistically homogeneous and isotropic. This implies that the 3PCF depends on three arguments only, which define the shape and size of a triangle. Let $\boldsymbol{X}_{i}, i=\{1,2,3\}$, be three points defining a triangle, and let $\boldsymbol{x}_{1}=\boldsymbol{X}_{3}-\boldsymbol{X}_{2}, \boldsymbol{x}_{2}=\boldsymbol{X}_{1}-\boldsymbol{X}_{3}$, and $\boldsymbol{x}_{3}=\boldsymbol{X}_{2}-\boldsymbol{X}_{1}$ be the side vectors. Furthermore, let $\gamma_{\mu}\left(\boldsymbol{X}_{i}\right)$, $\mu=\{1,2\}$, be the Cartesian components of the shear at the point $\boldsymbol{X}_{i}$. We note that the Cartesian components of the shear can also be considered as components of a symmetric trace-free matrix,

$\Gamma=\left(\begin{array}{cc}\gamma_{1} & \gamma_{2} \\ \gamma_{2} & -\gamma_{1}\end{array}\right)$,

and its behaviour under linear coordinate transformations $\boldsymbol{x} \rightarrow$ $\boldsymbol{x}^{\prime}=T \boldsymbol{x}$ is

$\Gamma^{\prime}=T^{\mathrm{T}} \Gamma T$.

Then, the Cartesian components of the 3PCF are defined as

$\gamma_{\mu \nu \lambda}\left(\boldsymbol{x}_{1}, \boldsymbol{x}_{2}, \boldsymbol{x}_{3}\right) \equiv\left\langle\gamma_{\mu}\left(\boldsymbol{X}_{1}\right) \gamma_{v}\left(\boldsymbol{X}_{2}\right) \gamma_{\lambda}\left(\boldsymbol{X}_{3}\right)\right\rangle$

where it was used that the shear field is a homogeneous random field, so that the $3 \mathrm{PCF}$ is invariant under translations; therefore, $\gamma_{\mu \nu \lambda}$ does depend only on the separation vectors $\boldsymbol{x}_{l}$. In order to employ the assumed rotational invariance of the shear field, one needs to measure the shear components relative to a direction that rotates with the triangle. For example, one can define the center of mass $\boldsymbol{o}=\left(\boldsymbol{X}_{1}+\boldsymbol{X}_{2}+\boldsymbol{X}_{3}\right) / 3$ of the triangle and define the shear components at $\boldsymbol{X}_{i}$ relative to the direction vector $\boldsymbol{X}_{i}-\boldsymbol{o}$ between the point $\boldsymbol{X}_{i}$ and the center $\boldsymbol{o}$. If $\zeta_{i}$ denotes the polar angle of the direction vector $\boldsymbol{X}_{i}-\boldsymbol{o}$, we define the tangential and cross component of the shear at $\boldsymbol{X}_{i}$ relative to the center $\boldsymbol{o}$ by

$$
\begin{aligned}
\gamma\left(\boldsymbol{X}_{i}, \zeta_{i}\right) & \equiv \gamma_{\mathrm{t}}\left(\boldsymbol{X}_{i}, \zeta_{i}\right)+\mathrm{i} \gamma_{\times}\left(\boldsymbol{X}_{i}, \zeta_{i}\right):=-\gamma\left(\boldsymbol{X}_{i}\right) \mathrm{e}^{-2 \mathrm{i} \zeta_{i}} \\
& =-\left[\gamma_{1}\left(\boldsymbol{X}_{i}\right)+\mathrm{i} \gamma_{2}\left(\boldsymbol{X}_{i}\right)\right] \mathrm{e}^{-2 \mathrm{i} \zeta_{i}}
\end{aligned}
$$

as can be seen from (1) when setting $T$ as a rotation matrix. We then define the components of the 3PCF relative to the center of mass as

$\gamma_{\mu \nu \lambda}^{(\mathrm{cen})}\left(x_{1}, x_{2}, x_{3}\right)=\left\langle\gamma_{\mu}\left(\boldsymbol{X}_{1}\right) \gamma_{\nu}\left(\boldsymbol{X}_{2}\right) \gamma_{\lambda}\left(\boldsymbol{X}_{3}\right)\right\rangle$, where now $\mu, v, \lambda=\{\mathrm{t}, \times\}$. It should be noted that the 3PCF defined in this way is invariant under rotations of the triangle, and thus depends only on the lengths $x_{i}=\left|x_{i}\right|$ of the side vectors, provided that we fix the orientation such that $\boldsymbol{x}_{1} \times \boldsymbol{x}_{2}=$ $x_{2} \times x_{3}=x_{3} \times x_{1}>0$.

There is nothing special about the center of mass; instead, different centers of the triangle (or other points attached to a triangle) can be chosen in the definition of the tangential and cross components of the shear, and thus in defining the 3PCF; SL03 considered some of the most obvious choices (orthocenter, center of incircle, center of circumcircle), and derived the relations between the $3 \mathrm{PCF}$ defined with respect to different centers.

\subsection{Parity transformation}

SL03 have derived the behavior of the 3PCF under parity transformations. Consider a fixed triangle, and its mirror symmetric sister obtained by flipping the original one along the line perpendicular to the side vector $\boldsymbol{x}_{3}$ going through $\boldsymbol{X}_{3}$. This transformation has two effects: first, the points $\boldsymbol{X}_{1}$ and $\boldsymbol{X}_{2}$ are interchanged, so that the orientation of the triangle is now negative, in the sense that $\boldsymbol{x}_{1} \times \boldsymbol{x}_{2}<0$. Second, the tangential components of the shears are unaffected, whereas the cross components change sign. Together, this implies for the behavior under parity transformations that

$\mathrm{P}\left[\gamma_{\mu \nu \lambda}^{(\mathrm{cen})}\left(x_{1}, x_{2}, x_{3}\right)\right]=\Pi \gamma_{\nu \mu \lambda}^{(\mathrm{cen})}\left(x_{2}, x_{1}, x_{3}\right)$,

where $\Pi$ is the parity; it is +1 if all of the indices of $\gamma$ are t's, or two $\times$ 's occur, otherwise it is -1 . Those compoents of the shear 3PCF for which $\Pi=+1$ are called even, the others are the odd components. Note the interchange of the arguments in (6). Furthermore, (6) applies equally well to the 3PCF of the shear if measured with respect to a different reference point attached to the triangle.

We can restate this result in the notation of Takada \& Jain (2003a,b). Instead of specifying a triangle by its side lengths, one can also characterize it by two sides and one angle. Let $\psi$ be the angle at the vertex $\boldsymbol{X}_{3}$ between the directions to $\boldsymbol{X}_{1}$ and $\boldsymbol{X}_{2}$, where $\psi \in[0, \pi]$ if $\boldsymbol{x}_{1} \times \boldsymbol{x}_{2}>0$, and $\psi \in[\pi, 2 \pi]$ otherwise. Then, $\gamma_{\mu \nu \lambda}\left(x_{1}, x_{2}, \psi\right)=\gamma_{\mu \nu \lambda}\left(x_{1}, x_{2}, x_{3}\right)$ for $\psi \in[0, \pi]$, and $\gamma_{\mu \nu \lambda}\left(x_{1}, x_{2}, \psi\right)=\gamma_{\nu \mu \lambda}\left(x_{2}, x_{1}, x_{3}\right)$ for $\psi \in[\pi, 2 \pi]$ in the new notation, and the parity transformation (6) becomes

$\mathrm{P}\left[\gamma_{\mu \nu \lambda}\left(x_{1}, x_{2}, \psi\right)\right]=\Pi \gamma_{\mu \nu \lambda}\left(x_{1}, x_{2}, 2 \pi-\psi\right)$.

\section{E- and B-modes of the shear}

Provided the shear field is due to gravitational lensing by a (geometrically-thin) matter distribution, it can be derived from a deflection potential $\psi$, according to

$\gamma=\gamma_{1}+\mathrm{i} \gamma_{2}=\frac{1}{2}\left(\psi_{, 11}-\psi_{, 22}\right)+\mathrm{i} \psi_{, 12}$,

where indices separated by a comma denote partial derivatives with respect to the Cartesian coordinates. A shear field with the property (8) is called an $E$-mode field. A general shear field will not satisfy (8), i.e., will not be derivable from a single 
scalar potential. However, a general shear field can be written as (see Crittenden et al. 2002; Schneider et al. 2002; see also Bunn et al. 2003 for an $E / B$-mode decomposition of the CMB polarization)

$$
\begin{aligned}
\gamma & =\left[\frac{1}{2}\left(\psi_{, 11}^{E}-\psi_{, 22}^{E}\right)-\psi_{, 12}^{B}\right]+\mathrm{i}\left[\psi_{, 12}^{E}+\frac{1}{2}\left(\psi_{, 11}^{B}-\psi_{, 22}^{B}\right)\right] \\
& =\left(\gamma_{1}^{E}+\gamma_{1}^{B}\right)+\mathrm{i}\left(\gamma_{2}^{E}+\gamma_{2}^{B}\right),
\end{aligned}
$$

with the two potentials $\psi^{E, B}$, which are conveniently combined into a complex field

$\psi=\psi^{E}+\mathrm{i} \psi^{B}$.

One also defines the Laplacians of the potentials,

$\nabla^{2} \psi^{E, B}=2 \kappa^{E, B}$

in lensing, $\kappa^{E}$ is the convergence (or dimensionless surface mass density) of the deflector. In the case of a matter distribution extending between sources at high redshift and us, as for lensing by the large-scale mass distribution in the Universe, the geometrically-thin lens theory no longer applies; however, both analytical considerations (e.g., Bernardeau et al. 1997; Schneider et al. 1998; van Waerbeke et al. 1999), as well as numerical investigations (e.g., Jain et al. 2000) have shown that the cosmic shear field is very close to an $E$-mode field; the $B$-mode contributions coming from multiple deflections are suppressed by a large factor compared to the $E$-mode shear. Furthermore, a small $B$-mode contribution derives from the spatial clustering of source galaxies (Schneider et al. 2002), but again is very much smaller than the $E$-mode, except at very small angular scales. However, if the shear is due not only to lensing, but also to, e.g., intrinsic alignments of galaxies (e.g., Heavens et al. 2000; Crittenden et al. 2001; Croft \& Metzler 2000; Catelan et al. 2000), a non-vanishing $B$-mode can be expected. Significant $B$-mode contributions to the cosmic shear signal have been measured (e.g., van Waerbeke et al. 2001a, 2002; Hoekstra et al. 2002; Jarvis et al. 2003).

It should be noted that the parity transformation behavior (6) or (7) are valid for a general shear field; in the derivation, no assumption on the $E / B$-mode character of the shear field has been made. Also, we have not yet used the fact that the statistical properties of the shear field are invariant under parity transformation (i.e., parity-symmetric). Takada \& Jain (2003a,b) have argued that the transformation laws (6), (7) only apply for $E$-modes, and that the signs are changed for a pure $B$-mode field. Their argument shall be reproduced here:

Consider a pure $E$-mode field, for which (7) applies. This can be turned into a $B$-mode field by rotating all shears by 45 degrees; in this operation, $\gamma_{\mathrm{t}} \rightarrow \gamma_{\times}$, and $\gamma_{\times} \rightarrow-\gamma_{\mathrm{t}}$. Hence, what was $\gamma_{\mathrm{ttt}}$ for the $E$-field becomes $\gamma_{\mathrm{x} \times \mathrm{x}}$ for the $B$-mode field, implying that $\gamma_{\times x \times}$ does not change sign under a parity transformation. This argument, however, is incomplete: the parity-reversed $B$-mode shear field is obtained from the parity-reversed $E$-mode shear field by rotating the shear by -45 degrees, and this guarantees that (7) remains valid even for a pure $B$-mode field. As mentioned above, the derivation of (6) and (7) makes no assumption about the character of the shear field, but is derived purely from geometrical considerations (literally, by drawing triangles with shear sticks attached to the vertices, and flipping them).

\section{Parity-symmetric shear fields}

In this section we shall consider the consequences of paritysymmetry for the shear field, in particular with regards to the 3PCF. Consider first the example just mentioned: take a pure $E$-mode field, e.g., coming from cosmological ray-tracing simulation, and rotate all shears by 45 degrees, to obtain a pure $B$-mode field. In the original $E$-mode field, peaks and valleys do not occur symmetrically: high-density peaks are present, but no deep valleys, since the dimensionless density contrast $\delta$ is bounded from below by -1 , but clusters of galaxies have a high density contrast (which, by the way, is the reason why clusters can be detected by shear measurements, but voids can not). Hence, the shear field will have tangentially-oriented patterns around mass concentrations, with no corresponding radial shear patterns present. The $B$-field, therefore, will have strong shear patterns with one circulation, but no corresponding ones with the opposite circulation. In other words, the $B$-mode field would carry circulation information, it would be possible to distinguish between a right-handed and a left-handed shear field, which obviously violates parity-symmetry. Hence, the asymmetry between peaks and valleys in the convergence field $\kappa^{E}$ from which the $E$-mode shear field is derived translates into an asymmetry between handedness of the $B$-mode field after the 45 degree rotation. If the convergence field would be symmetric, with as many peaks as valleys, or more precisely, if all odd moments of $\kappa$ would vanish (as is the case for a Gaussian random field), then this handedness problem in the $B$-mode field would not occur, and it would be paritysymmetric. What we shall show in this section is, that all correlation functions of the form

$\left\langle\gamma_{\mu}^{B}\left(\boldsymbol{X}_{1}\right) \ldots \gamma_{v}^{B}\left(\boldsymbol{X}_{m}\right) \gamma_{\lambda}^{E}\left(\boldsymbol{X}_{m+1}\right) \ldots \gamma_{\tau}^{E}\left(\boldsymbol{X}_{n}\right)\right\rangle \equiv 0$,

if $m$ is odd.

\subsection{Parity transformation of E/B-mode shear fields}

Consider a general shear field, which can be a combination of $E$ - and $B$-modes. Assume that we flip the field along a line through the origin, which encloses an angle $\zeta$ with the positive $x_{1}$-axis. A point $\boldsymbol{x}$ is mapped through this flipping to the point $\boldsymbol{x}^{\prime}=A \boldsymbol{x}$, where the matrix $A$ has the properties that $\operatorname{det} A=-1$, and $A A=1$ is the unit matrix. This second property shows that

$\boldsymbol{x}^{\prime}=A \boldsymbol{x} ; \boldsymbol{x}=A \boldsymbol{x}^{\prime}$

Explicitly,

$A=\left(\begin{array}{cc}\cos 2 \zeta & \sin 2 \zeta \\ \sin 2 \zeta & -\cos 2 \zeta\end{array}\right)$.

The parity-reversed shear field $\gamma^{\prime}\left(\boldsymbol{x}^{\prime}\right) \equiv(\mathrm{P} \gamma)\left(\boldsymbol{x}^{\prime}\right)$ can be derived as follows: let the shear at position $\boldsymbol{x}$ be $\gamma(\boldsymbol{x})=|\gamma| \mathrm{e}^{2 \mathrm{i} \varphi}$, where $\varphi$ is the angle the shear "stick" encloses with the positive $x_{1}$-axis; 
the flipped shear is then oriented in the direction $\varphi^{\prime}$ which satisfies $\varphi+\varphi^{\prime}=2 \zeta$, so that

$\gamma^{\prime}\left(\boldsymbol{x}^{\prime}\right)=|\gamma| \mathrm{e}^{2 \mathrm{i}(2 \zeta-\varphi)}=\mathrm{e}^{4 \mathrm{i} \zeta} \gamma^{*}(\boldsymbol{x})$.

The same result can of course be directly obtained from (1), setting $T=A$.

The shear field $\gamma^{\prime}$ will again be derivable from two fields $\psi^{E, B^{\prime}}$, for which the relation (9) is valid. Hence,

$\gamma_{1}^{\prime}\left(\boldsymbol{x}^{\prime}\right)=\frac{1}{2}\left(\frac{\partial^{2}}{\partial x_{1}^{\prime 2}}-\frac{\partial^{2}}{\partial x_{2}^{\prime 2}}\right) \psi^{E \prime}\left(\boldsymbol{x}^{\prime}\right)-\frac{\partial^{2}}{\partial x_{1}^{\prime} \partial x_{2}^{\prime}} \psi^{B^{\prime}}\left(\boldsymbol{x}^{\prime}\right)$

$\gamma_{2}^{\prime}\left(\boldsymbol{x}^{\prime}\right)=\frac{1}{2}\left(\frac{\partial^{2}}{\partial x_{1}^{\prime 2}}-\frac{\partial^{2}}{\partial x_{2}^{\prime 2}}\right) \psi^{B \prime}\left(\boldsymbol{x}^{\prime}\right)+\frac{\partial^{2}}{\partial x_{1}^{\prime} \partial x_{2}^{\prime}} \psi^{E \prime}\left(\boldsymbol{x}^{\prime}\right)$

The fields $\psi^{E, B \prime}$ will be related to the original ones by

$\psi^{E \prime}\left(\boldsymbol{x}^{\prime}\right)=\pi^{E} \psi^{E}(\boldsymbol{x}) ; \psi^{B \prime}\left(\boldsymbol{x}^{\prime}\right)=\pi^{B} \psi^{B}(\boldsymbol{x})$,

where the factors $\pi^{E, B}= \pm 1$ account for the possibility that the $\psi$ are not scalar fields, but pseudo-scalars. Introducing (17) into (15) and using

$\frac{\partial}{\partial x_{i}^{\prime}} \psi(\boldsymbol{x})=\frac{\partial x_{j}}{\partial x_{i}^{\prime}} \psi_{, j}(\boldsymbol{x})=A_{j i} \psi_{, j}(\boldsymbol{x})$,

where summation over repeated indices is implied and use has been made of (13), one finds

$\gamma_{1}^{\prime}\left(\boldsymbol{x}^{\prime}\right)=\frac{\pi^{E}}{2}\left(A_{i 1} A_{j 1}-A_{i 2} A_{j 2}\right) \psi_{, i j}^{E}-\pi^{B} A_{i 1} A_{j 2} \psi_{, i j}^{B}$,

$\gamma_{2}^{\prime}\left(\boldsymbol{x}^{\prime}\right)=\frac{\pi^{B}}{2}\left(A_{i 1} A_{j 1}-A_{i 2} A_{j 2}\right) \psi_{, i j}^{B}+\pi^{E} A_{i 1} A_{j 2} \psi_{, i j}^{E}$.

Using next the explicit representation (14) of the matrix $A$, one finds that

$\frac{\left(A_{i 1} A_{j 1}-A_{i 2} A_{j 2}\right)}{2} \psi_{, i j}=\frac{\psi_{, 11}-\psi_{, 22}}{2} \cos 4 \zeta+\psi_{, 12} \sin 4 \zeta ;$

$A_{i 1} A_{j 2} \psi_{, i j}=\frac{\psi_{, 11}-\psi_{, 22}}{2} \sin 4 \zeta-\psi_{, 12} \cos 4 \zeta$.

Using these relations in (18), one obtains

$$
\begin{aligned}
\gamma_{1}^{\prime}\left(\boldsymbol{x}^{\prime}\right)= & \left(\pi^{E} \frac{\psi_{, 11}^{E}-\psi_{, 22}^{E}}{2}+\pi^{B} \psi_{, 12}^{B}\right) \cos 4 \zeta \\
& +\left(\pi^{E} \psi_{,, 12}^{E}-\pi^{B} \frac{\psi_{, 11}^{B}-\psi_{, 22}^{B}}{2}\right) \sin 4 \zeta \\
= & \gamma_{1}(\boldsymbol{x}) \cos 4 \zeta+\gamma_{2}(\boldsymbol{x}) \sin 4 \zeta,
\end{aligned}
$$

where in the last step we have used the transformation law (15) of the shear. Similarly,

$$
\begin{aligned}
\gamma_{2}^{\prime}\left(\boldsymbol{x}^{\prime}\right)= & \left(\pi^{B} \frac{\psi_{, 11}^{B}-\psi_{, 22}^{B}}{2}-\pi^{E} \psi_{, 12}^{E}\right) \cos 4 \zeta \\
& +\left(\pi^{B} \psi_{,, 12}^{B}+\pi^{E} \frac{\psi_{, 11}^{E}-\psi_{, 22}^{E}}{2}\right) \sin 4 \zeta \\
= & \gamma_{1}(\boldsymbol{x}) \sin 4 \zeta-\gamma_{2}(\boldsymbol{x}) \cos 4 \zeta
\end{aligned}
$$

Comparing these last two equations with (9), one finds that $\pi^{E}=+1, \pi^{B}=-1$ : hence, the deflection potential of the $E$-mode field is a scalar field, as expected, whereas the potential of the $B$-mode field is a pseudo-scalar, so that

$\psi^{E \prime}\left(\boldsymbol{x}^{\prime}\right)=\psi^{E}(\boldsymbol{x}) ; \quad \psi^{B \prime}\left(\boldsymbol{x}^{\prime}\right)=-\psi^{B}(\boldsymbol{x})$,

or

$\psi^{\prime}\left(\boldsymbol{x}^{\prime}\right)=\psi^{*}(\boldsymbol{x})$

\subsection{Consequences for parity-symmetric shear fields}

If a shear field is parity-symmetric, the two fields $\gamma(\boldsymbol{x})$ and $\gamma^{\prime}(\boldsymbol{x})$ have the same statistical properties; in particular, all their correlation functions are identical. If one has a pure $B$-mode shear field, this then implies that all odd correlation functions vanish: from (22) one sees that the potential of the field $\gamma^{\prime}$ has the opposite sign of that of $\gamma$. Since the shear and the potential are linearly related, this implies that the signs of the two fields $\gamma$ and $\gamma^{\prime}$ are opposite, rendering all odd correlation functions zero. More generally, this argument shows that if we decompose the shear field into an $E$ - and $B$-field, as in (9), all correlations of the form

$\left\langle\gamma_{\mu}^{B}\left(\boldsymbol{X}_{1}\right) \gamma_{v}^{B}\left(\boldsymbol{X}_{2}\right) \gamma_{\lambda}^{B}\left(\boldsymbol{X}_{3}\right)\right\rangle=0 ;$

$\left\langle\gamma_{\mu}^{B}\left(\boldsymbol{X}_{1}\right) \gamma_{v}^{E}\left(\boldsymbol{X}_{2}\right) \gamma_{\lambda}^{E}\left(\boldsymbol{X}_{3}\right)\right\rangle=0$.

Therefore, only the pure $E$-field $3 \mathrm{PCF}$ and the mixed correlator $\left\langle\gamma_{\mu}^{B}\left(\boldsymbol{X}_{1}\right) \gamma_{v}^{B}\left(\boldsymbol{X}_{2}\right) \gamma_{\lambda}^{E}\left(\boldsymbol{X}_{3}\right)\right\rangle$

are non-zero for parity-symmetric fields. A further generalization then yields the result (12), namely that all correlators containing an odd number of $B$-mode shears must vanish. Furthermore, all odd correlation functions of $\kappa^{B}$ and $\psi^{B}$ vanish, for the same reason.

One example of this is well known from the 2PCF of the shear; the mixed correlator $\left\langle\gamma_{\mathrm{t}} \gamma_{\times}\right\rangle$vanishes identically due to parity invariance; on the other hand, this correlation function is linear in $\left\langle\kappa^{E} \kappa^{B}\right\rangle$ (see Schneider et al. 2002), and the result derived here applies.

\subsection{Aperture measures}

Given that all third order statistics of the shear are linearly related to the 3PCF, these results have further implications. Define the aperture measures for a point at the origin (Schneider 1996) as

$$
\begin{aligned}
& M_{\text {ap }}(\theta)=\int_{0}^{\theta} \mathrm{d}^{2} \vartheta Q(|\vartheta|) \gamma_{\mathrm{t}}(\vartheta) ; \\
& M_{\times}(\theta)=\int_{0}^{\theta} \mathrm{d}^{2} \vartheta Q(|\vartheta|) \gamma_{\times}(\vartheta),
\end{aligned}
$$

where the tangential and cross components of the shear are taken with respect tot he direction of the center of the circle of radius $\theta$, and $Q(\vartheta)$ is a weight function. These aperture measures have a number of very useful properties. Employing the 
relation (9) between shear and the potentials in Fourier space, it is easy to see that

$M_{\text {ap }}(\theta) \equiv 0$ for pure $B$-field

and

$M_{\times}(\theta) \equiv 0$ for pure $E$-field.

Therefore, these aperture measures cleanly separate $E$ - and $B$-modes; they are therefore used to obtain this separation in data fields, and essentially all detections of $B$-modes in secondorder cosmic shear statistics have been made using $\left\langle M_{\times}^{2}\right\rangle$ (van Waerbeke et al. 2001a, 2002; Hoekstra et al. 2002; Jarvis et al. 2003). Since $M_{\text {ap }}$ is a scalar quantity, it can be conveniently used to define higher-order cosmic shear statistics; in Schneider et al. (1998), the third-order statistics $\left\langle M_{\mathrm{ap}}^{3}\right\rangle$ was calculated using quasi-linear perturbation theory of structure growth, whereas van Waerbeke et al. (2001b) calculated $\left\langle M_{\mathrm{ap}}^{3}\right\rangle$ using the fitting formula of Scoccimarro \& Couchman (2001) for the non-linear evolution of the bispectrum of the cosmic density fluctuations. Munshi \& Coles (2003) derived this statistics using the hierarchical clustering ansatz, which is expected to give an accurate prescription of the cosmic density field on small scales.

$\left\langle M_{\mathrm{ap}}^{3}\right\rangle$ can be obtained from proper integration over the $E$-mode shear 3PCF $\left\langle\gamma_{\mu}^{E} \gamma_{\nu}^{E} \gamma_{\lambda}^{E}\right\rangle$; similarly, $\left\langle M_{\times}^{3}\right\rangle$ can be obtained by integrating over $\left\langle\gamma_{\mu}^{B} \gamma_{\nu}^{B} \gamma_{\lambda}^{B}\right\rangle$. The latter, however, vanishes identically for parity-symmetric shear fields, implying that $\left\langle M_{\times}^{3}\right\rangle=0$. For the same reason, $\left\langle M_{\mathrm{ap}}^{2} M_{\times}\right\rangle=0$. For the aperture measures, the handedness argument used above becomes even more intuitive.

Pen et al. (2003) employed third-order aperture statistics to the Virmos-Descart cosmic shear survey; they find a significant non-zero signal for all their four third-order moments. Their measured values of $\left\langle M_{\times}^{3}\right\rangle$ and $\left\langle M_{\mathrm{ap}}^{2} M_{\times}\right\rangle$shows that the data is not statistically parity invariant. Hence, non-zero values of these quantitites cannot be accounted for by intrinsic galaxy alignments, as their correlation functions are expected to be parity-symmetric as well, nor to higher-order lensing effects - again, they produce a parity-symmetric shear field. The remaining explanations are that the cosmic variance is larger than estimated by Pen et al., or that there is a yet undetected systematics in the data.

\section{Discussion}

We have shown that for a shear field whose statistical properties are invariant under parity transformation, all odd correlation functions of the $B$-mode shear vanish identically. This result has been derived by an explicit calculation of the properties of the parity-reversed shear field, as well as by a simple consideration: Whereas an asymmetry between peaks and valleys in the convergence $\kappa^{E}$, which leads to non-vanishing odd correlation functions of the $E$-mode shear field, signifies that matter overdensities and underdensities behave differently, a similar asymmetry in the corresponding field $\kappa^{B}$ yields an asymmetry between the two circularizations of the $B$-mode shear field, which for a parity-symmetric field is not permitted. In particular, the $3 \mathrm{PCF}$ of the shear vanishes identically for a pure $B$-mode field. This result is at variance to some claims in the literature, and we have attempted to explain the reasons for this.

One possibility to numerically generate a $B$-mode field which is parity-symmetric is the following: take a pure $E$-mode field, as it is obtained from ray-tracing simulations, and rotate all shears by 45 degrees. Take a second realization of the same random field (i.e., a second ray-tracing simulation with identical parameters but different random number seed), rotate by 45 degrees and parity flip the shear, or, equivalently, rotate the $E$-mode field by -45 degrees. Then take the average of these two fields, which is then a pure $B$-mode field and parity symmetric (by construction).

The result obtained here provides a very useful diagnostics for potential systematics in cosmic shear data. Whereas real $B$-modes can be present in the data, as they can be generated by intrinsic galaxy alignments or higher-order lensing effects, e.g. coming from source clustering, all these effects should obey parity symmetry. A significant detection of a correlation function involving an odd number of $B$-mode components, or a quantity derived from it (such as the aperture measures discussed above) is a sensitive probe of parity violation that probably can only be accounted for by systematics. It should be noted that a similar diagnostics has been used before for the 2PCF, namely checking that $\left\langle\gamma_{\mathrm{t}} \gamma_{\times}\right\rangle$vanishes.

Acknowledgements. I would like to thank M. Kilbinger, B. Jain, M. Lombardi, and M. Takada for very stimulating and helpful discussions. This work was supported by the German Ministry for Science and Education (BMBF) through the DLR under the project 50 OR 0106.

\section{References}

Bacon, D. J., Refregier, A. R., \& Ellis, R. S. 2000, MNRAS, 318, 625 Bartelmann, M., \& Schneider, P. 2001, Phys. Rep., 340, 291 (BS01)

Bernardeau, F., Mellier, Y., \& van Waerbeke, L. 2002, A\&A, 389, L28

Bernardeau, F., van Waerbeke, L., \& Mellier, Y. 1997, A\&A, 322, 1

Bernardeau, F., van Waerbeke, L., \& Mellier, Y. 2003, A\&A, 397, 405

Blandford, R. D., Saust, A. B., Brainerd, T. G., \& Villumsen, J. V. 1991, MNRAS, 251, 600

Bunn, E. F., Zaldarriaga, M., Tegmark, M., \& de Oliveira-Costa, A. 2003, Phys. Rev. D, 67, 023501

Catelan, P., Kamionkowski, M., \& Blandford, R. D. 2001, MNRAS, 320, L7

Crittenden, R. G., Natarajan, P., Pen, U.-L., \& Theuns, T. 2001, ApJ, 559,552

Crittenden, R. G., Natarajan, P., Pen, U.-L., \& Theuns, T. 2002, ApJ, 568,20

Croft, R. A. C., \& Metzler, C. A. 2000, ApJ, 545, 561

Heavens, A. F., Refregier, A., \& Heymans, C. E. C. 2000, MNRAS, 319, 649

Hoekstra, H., Yee, H. K. C., \& Gladders, M. D. 2002, ApJ, 577, 595

Jain, B., \& Seljak, U. 1997, ApJ, 484, 560

Jain, B., Seljak, U., \& White, S. D. M. 2000, ApJ, 530, 547

Jarvis, M., Bernstein, G. M., Fischer, P., et al. 2003, AJ, 125, 1014

Kaiser, N. 1992, ApJ, 388, 272

Kaiser, N., Wilson, G., \& Luppino, G. 2000 [astro-ph/0003338] 
Mellier, Y. 1999, ARA\&A, 37, 127

Miralda-Escudé, J. 1991, ApJ, 380, 1

Munshi, D., \& Coles, P. 2003, MNRAS, 338, 846

Pen, U.-L., Zhang, T., van Waerbeke, L., et al. 2003, ApJ, 592, 664

Schneider, P. 1996, MNRAS, 283, 837

Schneider, P., \& Lombardi, M. 2003, A\&A, 397, 809 (SL03)

Schneider, P., van Waerbeke, L., \& Mellier, Y. 2002, A\&A, 389, 729

Schneider, P., van Waerbeke, L., Jain, B., \& Kruse, G. 1998, MNRAS, 296, 873 (SvWJK)

Scoccimarro, R., \& Couchman, H. M. P. 2001, MNRAS, 325, 1312

Takada, M., \& Jain, B. 2003a, ApJ, 583, L49
Takada, M., \& Jain, B. 2003b [astro-ph/0304034]

van Waerbeke, L., Bernardeau, F., \& Mellier, Y. 1999, A\&A, 243, 15

Van Waerbeke, L., Mellier, Y., Erben, T., et al. 2000, A\&A, 358, 30

Van Waerbeke, L., Mellier, Y., Radovich, M., et al. 2001a, A\&A, 374, 757

Van Waerbeke, L., Hamana, T., Scoccimarro, R., Colombi, S., \& Bernardeau, F. 2001b, MNRAS, 322, 918

van Waerbeke, L., Mellier, Y., Pello, R., et al. 2002, A\&A, 393, 369

Wittman, D. M., Tyson, J. A., Kirkman, D., Dell'Antonio, I., \& Bernstein, G. 2000, Nature, 405, 143

Zaldarriaga, M., \& Scoccimarro, R. 2003, ApJ, 584, 559 